\title{
Die Frage nach der Gerechtigkeit im Rentensystem
}

\author{
Tim Köhler-Rama
}

\section{Vorbemerkung}

Das staatliche Rentensystem ist - wie die Sozialversicherung insgesamt eine Errungenschaft des Industrieproletariats. Als Reaktion auf die Arbeiterbewegung und das Erstarken der SPD wurde das Rentensystem Ende des 19. Jahrhunderts vom Staat implementiert, weil klar geworden war, dass die Reproduktionsbedingungen des Industriekapitalismus unerträglich geworden waren. Das Rentensystem sollte vor allem den Arbeiter vor allzu großer Not bewahren, dessen Arbeitskraft im Produktionsprozess verbraucht worden war. In aller Regel war die Rente zu dieser Zeit eine Invalidenrente. Altersrenten waren die Ausnahme. Wenn jemand hart gearbeitet und dabei seine Gesundheit ruiniert hatte, sollte er besser gestellt werden, als derjenige Fürsorgeempfänger, der nicht gearbeitet hat. Der Verbrauch der Arbeitskraft als Bedingung für eine Rente bildete somit den eigentlichen Grundgedanken des Rentensystems zum Zeitpunkt seiner Entstehung. Erst viel später, während des massiven Ausbaus des Rentensystems in den 1950er Jahren, wurde die Altersrente zur Norm und der Gedanke der Statussicherung im Alter („Rente als fortgesetzter Lohn“) überlagerte von nun an den Gedanken der Armutsvermeidung. Historisch gesehen enthält das Rentensystem somit die beiden Ziele Armutsvermeidung und Lebensstandardsicherung. Der folgende Beitrag soll zeigen, dass zwischen den beiden Zielen grundsätzlich eine Konkurrenz besteht, und der jüngste Anstieg der Altersarmut diese Zielkonkurrenz verschärft und daher auch die Frage nach der Gerechtigkeit im Rentensystem neu aufkommen lässt. Unmittelbar daran geknüpft ist die Frage, welche Rolle Umverteilung und Äquivalenz in der Ausgestaltung des Rentensystems spielen. Letztlich plädiert der Autor für ein gerechteres Rentensystem, das mithilfe von mehr Umverteilung innerhalb der Versichertengemeinschaft das Altersarmutsrisiko reduziert und dabei trotzdem als zentrales Institut zur Eigenvorsorge der Arbeitnehmerinnen und Arbeitnehmer erhalten bleibt. 


\section{Welches Ziel verfolgt das staatliche Rentensystem?}

Die Beantwortung der Frage, welches Ziel das Rentensystem in Deutschland prioritär verfolgen soll, hängt davon $\mathrm{ab}$, welche Gerechtigkeitsvorstellung die hiesige Gesellschaft mit dem Rentensystem vorrangig verknüpft. Letztlich muss in der Gesellschaft eine Einigung über die Frage erzielt werden, welche Art der Gerechtigkeit das Rentensystem produzieren soll. Oder anders formuliert: Es muss Einigkeit über die Frage erzielt werden, was eine gerechte Rente ist.

Was ist ein gerechtes Gesundheitssystem? Diese Frage ist einfach und wenig kontrovers zu beantworten. Jeder soll - unabhängig von seinem Einkommen - Zugang zu den notwendigen medizinischen Leistungen erhalten. Der Beitragssatz zur gesetzlichen Krankenversicherung ist für alle abhängig Beschäftigten gleich, d. h. er bemisst sich unabhängig vom individuellen Krankheitsrisiko. Im Ergebnis zahlt der Gutverdiener mehr Geld für denselben Versicherungsumfang, für denselben Leistungskatalog im Bedarfsfall als der Geringverdiener. Dass dies gerecht ist, wird mehrheitlich so gesehen. Offenbar besteht hierzulande also ein breiter gesellschaftlicher Konsens dahingehend, dass das Gesundheitssystem und das System der gesetzlichen Krankenversicherung dazu beitragen sollen, für mehr Bedarfsgerechtigkeit zu sorgen.

Anders beim Thema Rente. Hier besteht kein Konsens bezüglich der Frage, ob das Rentensystem vor allem mehr soziale Gerechtigkeit schaffen soll oder ob es vor allem dem Grundprinzip der Leistungsgerechtigkeit verpflichtet sein soll. Im derzeit herrschenden Rentensystem bezahlt jeder Versicherte - analog zum System der gesetzlichen Krankenversicherung denselben Beitragssatz, unabhängig von der Einkommenshöhe und unabhängig von dem individuellen Langlebigkeits- und Invaliditätsrisiko. Für denselben Beitragssatz bekommen aber - anders als im Gesundheitssystem - nicht alle Versicherten dieselbe Leistung im Falle des Risikoeintritts. Der Gutverdiener bezahlt im Rentensystem - anders als im Gesundheitssystem - eben nicht ein absolut höheres Beitragsvolumen als der Geringverdiener, um den Anspruch auf eine armutsvermeidende Rente zu erhalten. Jeder Versicherte muss unabbängig von seinem Einkommen derzeit 28 Entgeltpunkte auf seinem Rentenkonto ansammeln, um im Alter eine Rente zu bekommen, die so hoch ist wie die Grundsicherung, die jeder Bedürftige erhält, auch derjenige, der keinerlei Vorleistungen erbracht hat bzw. Beiträge in das System eingezahlt hat (vgl. Steffen 2019). Der Gutverdiener mit doppeltem Durchschnittsverdienst muss also rund 14 Jahre arbeiten, um im Alter die Sozialhilfeschwelle zu überschreiten. Der Geringverdiener mit halbem Durchschnittsverdienst (2019: rund 1.900 Euro monatlich) da- 
gegen erreicht die notwendigen 28 Entgeltpunkte rechnerisch erst nach 56 Jahren. Im Jahre 2003 waren hierfür noch 8 Jahre weniger notwendig. ${ }^{1}$ Im Ergebnis können es Menschen mit geringem Einkommen - und das sind hierzulande viele Millionen - im gegenwärtigen Rentensystem aufgrund des stark ausgeprägten Beitragsbezugs der Rentenhöhe (Äquivalenz) also nicht schaffen, im Alter eine Rente zu erhalten, die höher ist als die Alterssicherung einer Person, die keine Vorsorge betrieben hat und im Alter deshalb auf staatliche Fürsorgeleistungen angewiesen ist. Die Tatsache, dass das Rentensystem in Deutschland als eines der wenigen Länder innerhalb der OECD keine systematische Aufwertung geringer Einkommen bei der Rentenberechnung vornimmt (vgl. OECD 2019, S. 146ff.), wird hierzulande von vielen Menschen (und Rentenexperten!) für gerecht gehalten. Das bedeutet im Ergebnis, dass die Leistungsgerechtigkeit als Gerechtigkeitsvorstellung hierzulande im Zusammenhang mit dem Rentensystem tatsächlich höher gewichtet wird als das Ziel einer adäquaten Absicherung benachteiligter Menschen, die außerdem einem relativ hohen Invaliditätsrisiko und als Folge davon auch einem relativ hohen Sterblichkeitsrisiko ausgesetzt sind. Die Tatsache nämlich, dass die individuelle Risikolage beim Beitragssatz und bei der Rentenberechnung nicht berücksichtigt wird, benachteiligt im Ergebnis Personen mit geringem sozialen Status doppelt: Sie sind aufgrund ihres geringen Verdienstes nicht nur einem besonders hohen Altersarmutsrisiko ausgesetzt, sondern sie erhalten ihre geringen Renten auch im Durchschnitt deutlich kürzer als Personen mit hohem sozialen Status. ${ }^{2}$

Diese Prädominanz des Leistungsgedankens in Bezug auf das System der gesetzlichen Rentenversicherung in Deutschland ist irritierend, nicht zuletzt deshalb, weil diese Gerechtigkeitsvorstellung so stark abweicht von denen, die in den anderen Sozialversicherungszweigen vorherrschen. Im Rentensystem gilt hierzulande bislang: Nicht das Ziel, dass Geringverdiener, Frauen in Teilzeitbeschäftigung und körperlich schwer arbeitende Menschen im Alter nicht arm sind, ist prioritär, sondern die Realisierung des Prinzips, dass die Rente einer Person, die im Laufe ihres Arbeitslebens doppelt so viel verdient hat wie eine andere Person, exakt doppelt so hoch

1 Johannes Steffen spricht in diesem Zusammenhang von einem „Prozess systemischer Verschmelzung“ der Renten- und Grundsicherungssysteme, s. Steffen 2019.

2 Menschen aus den unteren Lohngruppen erhalten aufgrund ihrer geringen Lebenserwartung überproportional weniger Rentenzahlungen im Verhältnis zu den eingezahlten Beiträgen. Und der Abstand bei den Lebenserwartungen zu den Besserverdienenden hat in den letzten Jahren weiter zugenommen. Vgl. Haan, Peter et al. 2019. 
wie die Rente des anderen ausfällt. Dieses Prinzip wird als Äquivalenzprinzip bezeichnet und lehnt sich im Grunde an die Idee der Vermögensbildung im Bereich der kapitalfundierten Altersvorsorge an: Wer lange und viel spart, soll am Ende möglichst viel herausbekommen. Die Stringenz, mit der dieses Prinzip für das Rentensystem als das grundlegende Prinzip postuliert wird, ist allerdings deplatziert. Denn das staatliche Rentensystem dient - wie die Sozialversicherung insgesamt - vor allem den abhängig Beschäftigten, die kaum über weitere Ressourcen verfügen als ihre eigene Arbeitskraft, die sie tagtäglich verwerten und reproduzieren müssen. Hierzu gehört auch die Absicherung gegen große Lebensrisiken im Rahmen der Sozialversicherung. Wenn das Arbeitseinkommen in immer mehr Fällen nicht (mehr) ausreicht, um Armut im Alter zu vermeiden, wird das Beharren auf dem Äquivalenzprinzip im Rentensystem in der sozialpolitischen Debatte zunehmend kontraproduktiv, weil es effektiven Reformvorschlägen entgegensteht. Für denjenigen Versicherten, der im Alter trotz langjähriger Beitragszahlungen arm ist, nützt es nichts, wenn ihm gesagt wird, dass das Grundprinzip der Äquivalenz im Rentensystem bislang unbeschadet geblieben ist. Die Vermeidung von Armut für die Fälle, in denen die Verwertung und Reproduktion der Arbeitskraft nicht mehr möglich sind, stellt die eigentliche Essenz der Sozialversicherung dar. Für das Rentensystem heißt das: Wenn die Altersarmut stark zunimmt - wie das heute wieder der Fall ist -, müssen die armutsvermeidenden Elemente und die Mechanismen des sozialen Ausgleichs im Rentenrecht (wieder) gestärkt werden. Das Rentensystem ist keine Sparinstitut, sondern eine soziale Risikoversicherung.

So scheint es zunehmend zweifelhaft, dass sich das Ziel der Statussicherung im Rentensystem (mithilfe des Instruments der Äquivalenz) angesichts einer sich stark veränderten sozioökonomischen Kontextsituation seit Anfang der 2000er Jahre auch künftig weiterhin höher gewichten lässt als das Ziel der Armutsvermeidung. Die Armutsgefährdungsquote der über 65jährigen Personen ist (laut Mikrozensus) im Zeitraum zwischen 2005 und 2018 von $11 \%$ auf knapp 14,7\% angestiegen (BIAJ 2019). Andere Daten weisen höhere Armutsquoten aus. Vor allem bei den Männern in Ostdeutschland ist seit einigen Jahren ein sehr starker Rückgang der durchschnittlichen Rentenansprüche bei den jüngeren Geburtsjahrgängen zu konstatieren. Für diese Gruppe wird vom Deutschen Institut für Wirtschaftsforschung (DIW) ein Anstieg der Altersarmut bis 2030 auf $36 \%$ erwartet (Haan et al. 2017). Zentrale Gründe für diese Entwicklung sind die aufgrund der hohen Arbeitslosigkeit der 1990er Jahre und früheren 2000er Jahre zunehmend schlechten Erwerbsbiografien in Ostdeutschland. Je weniger soziale Risikolagen wie Ausbildung und Arbeitslosigkeit bei der Ren- 
tenberechnung berücksichtigt werden, desto weniger Einkommenssicherheit im Alter bietet das Rentensystem. ${ }^{3}$ Die immer stärkere Lohnspreizung und die gestiegene Bedeutung des Niedriglohnsektors wirken immer stärker darauf hin, dass das Ziel der Armutsvermeidung mittels einer stärkeren Berücksichtigung sozialer Risikolagen und einer systematischen Aufwertung geringer Einkommen bei der Rentenberechnung nach ganz oben auf die rentenpolitische Agenda rückt. Die lange Diskussion über die (überfällige) Einführung einer - wie auch immer definierten - „Grundrente“ und über die Fortführung der Hochwertung geringer Einkommen bei der Rentenberechnung, die es seit 1972 für Zeiten bis 1992 im Rentenrecht gibt, sind Vorboten hierfür (Köhler-Rama 2019). Das in den 1950er Jahren eingeführte Rentensystem in seiner jetzigen Konzeption scheint sich jedenfalls überlebt zu haben, weil es zu vielen Versicherten zu wenig Schutz vor Altersarmut bietet. ${ }^{4}$

\section{Soll das staatliche Rentensystem Armut vermeiden?}

Die Tatsache, dass es hierzulande zu der Frage, ob das staatliche Rentensystem Armut vermeiden soll oder nicht, bislang keinen Konsens gibt, liegt möglicherweise daran, dass das Rentensystem im Grunde eine Art Zwittersystem ist, das seit über 60 Jahren zwei Ziele zugleich verfolgt: Die Armutsvermeidung und die Anerkennung von Lebensleistung. Die Realisierung beider Ziele zugleich hat viele Jahrzehnte lang zu einer hohen Akzeptanz des Rentensystems in Deutschland beigetragen. Für ein staatliches Rentensystem als Pflichtversicherungssystem, das auch ausreichend gegen Altersarmut schützt, spricht vor allem, dass die gesetzliche Rentenversicherung ein Zweig der Sozialversicherung ist, zu deren Wesen der soziale Ausgleich zwingend dazu gehört, weil gerade er sie von der Privatversicherung unterscheidet (vgl. Rolfs 2000). Armutsvermeidung lässt sich - wie eingangs er-

3 Insbesondere infolge der hohen Niedriglohnquoten in West- und Ostdeutschland (West: 22,7\%; Ost: $35 \%$ ), kommt es zu einem Rückgang der Rentenanwartschaften im unteren Einkommenssegment. Auch die Anzahl der Entgeltpunkte (EP) des Medianrentners - also des mittleren Rentners - geht sukzessive zurück. Während der ostdeutsche Medianrentner (Zugang) im Jahre 1993 noch 51 EP aufwies, waren es 2016 nur noch 39 EP. Der westdeutsche Medianrentner (Zugang) hatte 1993 noch 47 EP, 2016 waren es noch 44 EP. S. Himmelreicher 2018, S. 337.

4 Ein Beispiel für die Lebensfremdheit des Rentensystems: Der Durchschnittsverdienst einer Altenpflege-Fachkraft in Sachsen liegt aktuell bei rd. 2.200 Euro/mtl. Um auf die 28 EP (Nettorente: 800 Euro) zu kommen, muss sie 40 Jahre durchgehend Vollzeit (!) arbeiten. 
läutert - aus historischer Perspektive problemlos als ein originäres Ziel der gesetzlichen Rentenversicherung beschreiben. Außerdem ist die gesetzliche Rentenversicherung noch immer das einzige halbwegs universale Pflicht-Vorsorgesystem für abhängig Beschäftigte in Deutschland. Nur ihr kann daher im deutschen Sozialstaat die Aufgabe zugewiesen werden, Altersarmut zu vermeiden. Die einzige Alternative hierzu bestünde darin, das Thema Altersarmut dauerhaft - wie es bis zur Einführung des Rentensystems bis 1889 der Fall war - an das Fürsorgesystem (seit 2003 euphemistisch: „Grundsicherung im Alter") zu adressieren. Dies tun zwar viele, dagegen spricht aber ganz eindeutig, dass die Grundsicherung nicht armutsvermeidend ist, sondern lediglich das Existenzminimum sichert. Wenn massenhaft langjährig Versicherte und Vollzeit beschäftigte Menschen am Ende ihres Erwerbslebens absehbar im Fürsorgesystem landen, entwertet dies nicht nur ihre Lebensleistung (weil hier nur ihre Bedürftigkeit, nicht ihre Versicherungsbiografie, geprüft wird), sondern eine Pflichtversicherung ist dann verfassungsrechtlich im Grunde nicht mehr legitimierbar (vgl. Eichenhofer 2019). Die Lösung des Themas Altersarmut lässt sich daher unmöglich an das Grundsicherungssystem delegieren.

Gegen diese Argumentation wird angeführt, dass die gesetzliche Rentenversicherung lediglich eine Versicherung darstellt, die wegfallendes Einkommen im Alter ersetzt. Zuwenig Rente sei die Folge eines zu geringen Einkommens und schlechter Arbeitsmarktverhältnisse, mit deren Kompensation das Rentensystem überfordert wäre. Außerdem sei die Vermeidung von Altersarmut eine gesamtgesellschaftliche Aufgabe, deren Lösung im Rahmen des Steuersystems erfolgen solle. Das sei gerechter und effizienter. Der zielgenauen und effizienten Armutsvermeidung diene die Grundsicherung, weil hier die tatsächliche Bedürftigkeit im Haushaltskontext berücksichtigt werde. Wer im Alter nicht arm sein wolle, könne und müsse zusätzlich privat vorsorgen (vgl. Ruland 2012).

Diese Argumentation übersieht, dass Armutsvermeidung tatsächlich eine gesamtgesellschaftliche Aufgabe ist, zu deren Lösung allerdings gerade das Rentensystem eingeführt wurde. Das innovative Element der Einführung des Rentensystems bestand gerade darin, das staatliche Fürsorgesystem zu überwinden. Mit der Rentenreform von 1957 wurde das staatliche Rentensystem dann so konzipiert, dass ein langjährig Vollzeit tätiger Mensch (zu jener Zeit in der Regel ein Mann) nach einem erfüllten Arbeitsleben - damals hieß das 40 Versicherungsjahre - im Alter eine Rente erhält, die nicht nur ausreicht, Armut zu vermeiden, sondern seinen erreichten Lebensstandard zu halten. Seinerzeit wurde dies mit einem Bruttorentenniveau in Höhe von $60 \%$ assoziiert (vgl. Schmähl 2018, S. 239ff). Ähnlich wie heute gab es in den 1950er Jahren einen starken Strukturwan- 
del und als Reaktion darauf ein starkes Sicherheitsbedürfnis der Bevölkerung. Mit den Prinzipien Lohnersatz, Dynamisierung und Umlagefinanzierung kam die sehr populäre Rentenreform 1957 diesem Sicherheitsbedürfnis entgegen. Die Rentenreform von 1957 lässt sich daher auch als „Armutspolitik auf der Basis des Versicherungsprinzips“ (Göckenjan 2000, S. 373) beschreiben. Ohne diese Reform hätte der Personenkreis der Arbeitnehmer im Alter zwangsläufig zur Armutspopulation gezählt. Für diesen Personenkreis bedeutet das Konzept des Lohnersatzes, d.h. die Einkommenssicherung auf ungefähr dem Lebenshaltungsniveau, das im Erwerbsleben erreicht wurde, „nicht Belohnung oder Privilegierung, sondern schlicht Existenzsicherung" (Göckenjan 2000, S. 374).

Seit 1957 hatte die gesetzliche Rente somit eine existenzsichernde Lohnersatzfunktion. Das bedeutet, dass das Ziel der Armutsvermeidung von Beginn an implizit enthalten war. Wie wichtig das Ziel der Armutsvermeidung war, zeigt auch, dass die SPD bereits im Vorfeld der 1957er-Reform vorgeschlagen hatte, ein "fiktives Mindesteinkommen“ bei der Rentenberechnung vorzusehen. Voraussetzung sollte sein, dass die Hälfte der Zeit zwischen Beginn der Versicherung und dem Versicherungsfall mit „Beiträgen belegt" ist (vgl. Schmähl 2018, S. 249). In diesem Fall sollte bei der Rentenberechnung mindestens die Hälfte der allgemeinen Bemessungsgrundlage zugrunde gelegt werden. Eine solche Rente nach Mindesteinkommen wurde dann allerdings erst 1972 tatsächlich in das Rentenrecht eingeführt.

\section{Die Entwicklung der Rente: Vom „Einkommenszuschuss“ zur "Leistungsrente"}

Ursprünglich diente wurde - wie eingangs erwähnt - die gesetzliche Rentenversicherung Ende des 19. Jahrhunderts primär als eine Versicherung gegen die Folgen des Invaliditätsrisikos eingeführt. Die Altersgrenze lag seinerzeit bei 70 Jahren. Zugleich lag das durchschnittliche Rentenzugangsalter bis zur Jahrhundertwende bei 56 Jahren (vgl. Torp 2019, S. 134). Da die Altersgrenze nur von wenigen Menschen erreicht wurde, diente die Rentenleistung während der ersten Jahrzehnte nach Einführung des Rentensystems vor allem der Abmilderung der Folgen von Invalidität. Die Rentenformel bestand zum einen aus einem Grundbetrag und zum anderen aus einem beitragszeit- und einkommensabhängigen Steigerungsbetrag. Die Spreizung der Rentenleistungen war gering. 1890 lag der Durchschnittszahlbetrag der Invalidenrente bei 140 Mark, was etwa $20 \%$ des 
durchschnittlichen Bruttolohns entsprach. ${ }^{5}$ Der Grundgedanke Otto von Bismarcks in Verbindung mit der Einführung der „Invaliditäts- und Altersversicherung" im Jahre 1889 war es, den Arbeitern einen Rechtsanspruch auf eine Rente als Gegenleistung für lebenslange Arbeit einzuräumen. „Bismarcks Idee bei der Einführung der Arbeiterversicherungen war es, dass das, was ein invalider und deshalb arbeitsunfähiger Arbeiter erhalten ,muss', als ein ihm zustehendes Recht konzipiert wird“ (Wallrabenstein 2009, S. 65-66). Die Invalidenrente war zwar zu gering, um Armut im Falle der Invalidität zu vermeiden, sie milderte aber immerhin die Folgen des Einkommensausfalls infolge der nachlassenden Erwerbsfähigkeit ab. Vor allem aber verbesserte sie die Rechtsposition des mittellosen abhängig Beschäftigten in einer Zeit immer noch brutaler Ausbeutung der Arbeitskraft während des expandierenden Industriekapitalismus.

Die nächste große Zäsur des gesetzlichen Rentensystems brachte die bereits erwähnte Rentenreform von 1957. Mit der während der Kanzlerschaft Konrad Adenauers eingeführten "dynamischen Rente" wuchs das Leistungsniveau der sozialen Sicherung erstmals deutlich über die Bedarfsgrenze des Existenzminimums hinaus. Die gesetzliche Rente sollte fortan nicht mehr nur einen Zuschuss zum Lebensunterhalt, sondern einen echten Lohnersatz darstellen. Als neues Ziel rückte - vor dem Hintergrund starker Lohnzuwächse in der Zeit des „Wirtschaftswunders“ - die sozialpolitische Absicherung des erworbenen Lebensstandards und des relativen sozialen Status in den Fokus der Alterssicherungspolitik (vgl. Hockerts 1990, S. 93). Demgemäß wurde eine neue Rentenformel eingeführt. Der Grundbetrag entfiel. Stattdessen wurde zur Berechnung der Rente die durchschnittliche Lohnposition während des gesamten Erwerbsverlaufs die maßgebliche Berechnungsgröße. Auf diese Weise wurde das Versicherungsprinzip gestärkt. Die Rente bekam den Charakter einer „Leistungsrente“. Die Spreizung der Rentenzahlbeträge nahm zu. Am meisten profitierten von der Rentenreform die gutverdienenden Beschäftigten, weil von nun an nur noch die durchschnittliche individuelle Einkommensposition im Erwerbsverlauf die Höhe der Rente bestimmte. Zugleich wurde der Gedanke der Eigenvorsorge zum Leitgedanken des Rentensystems. Dies machte zum einen den Steueranteil an der Finanzierung der Rentenausga-

52018 lag die durchschnittliche Erwerbsminderungsrente bei 818 Euro, was rd. $26 \%$ des Durchschnittlohns im Jahre 2018 entsprach. Vgl. DRV Bund (2019, S. 14,36 . 
ben bis heute begründungsbedürftig. ${ }^{6}$ Zum anderen bedeutete dies vor allem eine Stärkung des Äquivalenz- und Leistungsgedankens im Rentensystem (vgl. Wallrabenstein 2009, S. 65-66).

Diese Grundprinzipien der Rentenreform 1957 prägen bis heute das öffentliche Rentensystem. Vermutlich aus diesem Grunde wird in Deutschland bis heute - anders als in vielen anderen Ländern - eine gerechte Rente vor allem mit einer leistungsgerechten Rente assoziiert. Als Folge davon wird eine stärkere Umverteilung innerhalb des Rentensystems mehrheitlich (noch immer) kritisch gesehen. Es ist allerdings fraglich, ob diese majoritäre Auffassung angesichts des stark ansteigenden Altersarmutsrisikos auch künftig Bestand haben wird. Die Geschichte zeigt, dass grassierende Altersarmut auf so viel Unzufriedenheit in der Bevölkerung stößt, dass in diesem Fall tiefgreifende sozialpolitische Reformen unumgänglich werden. Ähnlich wie zu der Zeit der Einführung des Rentensystems Ende des 19. Jahrhunderts, hatte auch die Rentenreform 1957 vor allem das Ziel, kurzfristig das Problem der massenhaften Altersarmut abzumildern. Historisch gesehen war somit das Äquivalenzprinzip immer vor allem ein Mittel zum Zweck. Es sollte die Aufwertung der Renten und deren Finanzierung in der Gesellschaft konsensfähig machen. Das wichtigste Element der Reform für die Menschen war allerdings die rd. 70 \%ige (!) Anhebung der laufenden Renten, was vor allem mit einer drastischen Anhebung des Beitragssatzes von $11 \%$ auf $14 \%$ finanziert wurde. Zur Durchsetzung dieser massiven Ausweitung des staatlichen Rentensystems gegen den vereinten Widerstand der Arbeitgeber, der Versicherungswirtschaft, der Bundesbank und dem Bundesfinanzministerium war die Proklamierung der Stärkung des Versicherungsprinzips unerlässlich. Anders formuliert: Ohne die die Stärkung des Äquivalenzprinzips wäre die Durchsetzung des eigentlichen Ziels der Reform, die unmittelbare Beseitigung und langfristige Vermeidung von Altersarmut, nicht mehrheitsfähig gewesen.

Das Rentensystem enthält seit 1957 somit implizit zwei Ziele - Armutsvermeidung und Statussicherung - und zugleich ein legitimations- und akzeptanzstiftendes Prinzip, nämlich das Äquivalenzprinzip. Das Problem, das sich aus dieser Melange ergibt, lässt sich als „Dualität“ oder „Gerechtigkeitsdilemma" beschreiben, denn die beiden Ziele stehen in gewisser Weise konträr zueinander. Effektive Armutsvermeidung bedingt soziale Ausgleichsmechanismen innerhalb des Systems im Sinne einer bedarfsgerechten Rente. Der zähe Streit um die Bedürftigkeitsprüfung im Zusammenhang

6 Im Rentensystem vor 1957 diente der Staatszuschuss der Finanzierung des einheitlichen Sockelbetrags der Rente. Dieser entfiel infolge der Rentenreform 1957. 
mit der Grundrente zeigt, dass hierfür bislang keine Mehrheit in Deutschland existiert. Statussicherung bedeutet dagegen eine leistungsgerechte Rente, der nach herrschender Auffassung eine Stärkung von Umverteilungselementen innerhalb des Rentensystems entgegensteht. Wenn das Rentensystem allerdings - ohne allgemeine Leistungsausweitung - das steigende Altersarmutsrisiko mindern soll, müssen interne Umverteilungsmechanismen gestärkt werden. Dies stellt einige Versicherte besser, andere schlechter. Das Beitrags-Leistungsverhältnis für die Begünstigten verbessert sich, für andere verschlechtert es sich. Tatsächlich steht dies dem Äquivalenzprinzip entgegen. Die meisten Experten und Beobachter assoziieren daher mit Umverteilungselementen im Rentensystem sogenannte „versicherungsfremde Leistungen“. Dabei übersehen sie, dass eine effektive Absicherung sozial sehr ungleich verteilter Risiken - und das Invaliditäts- und Langlebigkeitsrisiko gehören selbstverständlich hierzu - Ausgleichselemente unbedingt notwendig macht.

Mehr Armutsvermeidung bedeutet also zwingend mehr Umverteilung innerhalb des Rentensystems. Mehr Umverteilung bedeutet Verletzung des Äquivalenzprinzips. Zugleich setzt eine Statussicherung die Armutsvermeidung eigentlich voraus, denn ohne Armutsvermeidung macht Statussicherung keinen Sinn. Die Realisierung beider Ziele benötigt eine ausreichende finanzielle Basis des Rentensystems. Es ist klar: Eine Fortsetzung der Grundideen von 1957 bedingt in Zeiten des Anstiegs der Lebenserwartung und des sukzessiven Hineinwachsens geburtenstarker Jahrgänge in das Rentenalter einen Anstieg der Kosten der Alterssicherung. Das heißt: Die Gesellschaft insgesamt muss mehr Ressourcen aufwenden, um das steigende Langlebigkeitsrisiko und das steigende Invaliditätsrisiko ausreichend zu finanzieren.

\section{Soziale Ausgleichselemente im Rentensystem wurden geschwächt}

Das Rentensystem steht vor diesem Hintergrund heute also (wieder einmal) vor der Aufgabe, seine prioritäre Zielsetzung (und damit auch seine prioritären Funktionsprinzipien) klar zu definieren. Der Paradigmenwechsel 2001 mit der Einführung von bindenden Beitragssatzzielen (die inzwischen weit unterschritten wurden) und der Aufgabe eines eindeutigen Leistungsziels hat dazu geführt, dass die gesetzliche Rente zunehmend nur noch eine Art - immer häufiger auch so bezeichnete - „Basissicherung“ darstellt. Zugleich ist in der neuen Welt der niedrigen Zinsen die Idee, das sinkende Rentenniveau mithilfe zusätzlicher ausreichend sicherer Altersvorsorgeprodukte zu kompensieren, nachhaltig gescheitert. Immer dringli- 
cher stellt sich daher die Frage, welchen Weg das Rentensystem in Deutschland künftig gehen soll. Liegt die Zukunft des deutschen Rentensystems eher in der Adaption von Elementen des österreichischen Systems, das Anfang der 2000er Jahre die Teilprivatisierung des Rentensystems unterlassen hat und stattdessen das System in Richtung einer Bürgerversicherung weiter entwickelt hat (vgl. Blank et al. 2016)? Oder liegt die Zukunft eher in der Übernahme von Elementen des schwedischen Systems, das offenbar erfolgreich einige Vorteile kapitalgedeckter Systeme mit starker staatlicher Regulierung implementieren konnte? Eines ist aber ganz klar: Für eine Realisierung des Ziels der Vermeidung von Altersarmut bedarf es staatlich organisierter effektiver Umverteilungsmechanismen innerhalb des Rentensystems zugunsten vulnerabler Gruppen wie Erwerbsgeminderte, Geringverdiener (mit einer Überrepräsentanz von Frauen) und Langzeitarbeitslose. Das Problem ist, dass obgleich die sozialen Risiken bei den jüngeren Kohorten zugenommen haben - d.h. es gibt längere Ausbildungs- und Arbeitslosigkeitszeiten in den jüngeren Kohorten, mehr Zeiten mit niedrigen Verdiensten wegen Expansion des Niedriglohnsektors, mehr prekäre Beschäftigungsverhältnisse, sowie mehr und längere Phasen der Selbständigkeit (vgl. DRV Bund 2020) -, in den letzten 20 Jahren eine ganze Reihe sozialer Ausgleichselemente im Rentensystem gestrichen wurden, so u.a. die Reduzierung der rentenrechtlichen Bewertung von Schul- und Hochschulzeiten (vollständiger Wegfall der Bewertung seit 2009) und der vollständige Wegfall der Bewertung von Zeiten, in denen Arbeitslosengeld II bezogen wird (seit 2011). Die Konsequenzen dieser Maßnahmen sind ein sozial ungerechteres Rentensystem und weniger Einkommenssicherheit im Alter mit der Folge eines Anstiegs des Altersarmutsrisikos. Insgesamt wird so das Ziel der Armutsvermeidung immer weniger erfüllt. Zugleich fehlt - anders als in den meisten anderen EU-Staaten - in Deutschland noch immer eine ausreichende Mindestrente für langjährig Versicherte und eine systematische Hochwertung geringer Einkommen bei der Rentenberechnung. ${ }^{7}$ Die Rente nach Mindestentgeltpunkten (nur für Zeiten bis 1992) und die für 2021 angekündigte Grundrente (mit zu hohen $\mathrm{Zu}$ gangshürden) werden nicht ausreichen, um das System armutsfest zu ma-

7 Die derzeit noch systematische Hochwertung der Löhne in Ostdeutschland wird ab 2025 gänzlich entfallen. Das ist angesichts des besonders stark steigenden Altersarmutsrisikos in Ostdeutschland eine der vielen Paradoxien in der Rentenpolitik der letzten Jahre. 
chen. Insbesondere reichen diese Elemente nicht aus, die steigenden Verwertungsrisiken der Arbeitskraft in den immer prekärer werdenden Arbeitsmärkten zu kompensieren.

Begründet wurden die genannten Kürzungsmaßnahmen im Rentensystem durchweg (auch) mit dem Argument der Stärkung des Beitragsbezugs der Rente und der Stärkung der Leistungsgerechtigkeit. „Umverteilung gehört in das Steuersystem“, so die Argumentationslogik, die zu kurz greift. Tatsächlich gibt es im Rentensystem einen inhärenten Zielkonflikt zwischen Armutsvermeidung (mittels sozialer Ausgleichselemente) und Leistungsgerechtigkeit (mittels Stärkung des Beitrags-Leistungsbezugs). Allerdings zeigt der Blick in die Rentenhistorie, dass letztlich nur ein Rentensystem, dass die Bevölkerung als sozial- und leistungsgerecht einschätzt, gesellschaftlich akzeptiert wird. Die Rentenreform 1957 war deshalb so erfolgreich und hat der CDU - einmalig in ihrer Geschichte - ein absolute Mehrheit bei der Wahl 1957 verschafft, weil sie eine erfolgreiche „Armutspolitik auf der Basis des Versicherungsprinzips“ (Göckenjan 2000, S.737) einführte. Wenn man diese historische Erfahrung auf die aktuelle rentenpolitische Diskurslage übertragen möchte, heißt das, dass die Stärkung armutsvermeidender Elemente nur möglich ist, wenn der Rentenbeitrag angehoben wird und zugleich der Beitrags-Leistungsbezug nicht zu stark geschwächt wird. Das wiederum setzt einen tendenziell steigenden Anteil von Bundesmitteln an der Finanzierung der Rentenausgaben voraus. Um das zu begründen, benötigt man nicht die Verwendung des unscharfen Begriffs der sogenannten „versicherungsfremden Leistungen“, sondern es genügt der Gedanke der „Triparität“, der seit Bismarck de facto im Rentensystem realisiert wird. „Triparität“ bedeutet, dass der Bund der „dritte Beitragszahler" ist und für eine ausreichende Stabilität des Rentensystems sorgt. Er tut dies, weil die Gesellschaft insgesamt ein Interesse daran hat, dass das soziale Risiko der Altersarmut im Rahmen eines Pflichtversicherungssystems ausreichend versichert wird und auf diese Weise der soziale Friede im Land gewahrt bleibt und - als erwünschter Nebeneffekt - die Fürsorgesysteme entlastet werden. Mit diesen Argumenten setzte Otto von Bismarcks das Pflichtversicherungssystem erfolgreich gegen viele Widerstände der Versicherungsindustrie und der Arbeitgeber durch.

\section{Was tun, wenn Altersarmut wieder zu einem Massenphänomen wird?}

Aus der Rentengeschichte lässt sich vor allem lernen, dass die alleinige Adressierung des Altersarmutsrisikos an das Sozialhilfesystem keine Lösung ist, weil das staatliche Rentensystem in diesem Fall seine Akzeptanz 
und Legitimation verlieren würde. Es ist ganz einfach: Nach einem erfüllten Arbeitsleben muss eine Rente herauskommen, die oberhalb der Sozialhilfe liegt. Ansonsten erfüllt die (Pflicht-)Versicherung nicht ihren eigentlichen Zweck. Die Lösung in den meisten OECD-Staaten zur Sicherstellung dieses zentralen Grundgedankens liegt in der Hochwertung niedriger Einkommen bei der Rentenberechnung, d. h. höheren Ersatzraten für Geringverdiener. Das wird dort allgemein als gerecht empfunden, weil niedriges Einkommen im Niedriglohnsektor ein ungleich verteiltes soziales Risiko darstellt, was in der Regel sogar mit einem höheren Invaliditätsrisiko und einer kürzeren Lebenserwartung einhergeht. ${ }^{8}$ So lässt sich eine Stärkung sozialer Ausgleichselemente im Rentenrecht zugunsten von Menschen, die unter Lohndiskriminierung auf dem Arbeitsmarkt leiden, gut begründen. Einer Aufstockung geringer Arbeitsentgelte bei der Rentenberechnung liegt dabei eine ganz andere Konzeption zugrunde als eine gänzlich vorleistungsunabhängige Grundrente. „Basic pensions“ in den meisten der 18 OECD-Staaten, in denen eine solche existiert, kollidieren nicht grundsätzlich mit dem Prinzip der Beitragsäquivalenz, weil sie an die individuelle Vorleistung in Form von Beiträgen in das Rentensystem anknüpfen. Eine Mindestrente mit einer umfassenden Bedürftigkeitsprüfung unter Berücksichtigung des gesamten Haushaltes und sämtlicher Einkommensarten (Means-tested Minimum Pension) würde dagegen dem Grundgedanken der Rente als „Lohnersatz“ gänzlich entgegenstehen. In diesem Fall würde „der Rahmen des zulässigen sozialen Ausgleichs innerhalb der Versichertengemeinschaft verlassen und die Hinwendung zur Wahrnehmung einer Aufgabe öffentlicher Fürsorge (...) vollzogen“, schlussfolgert der Verfassungsrechtler Wallerath und hält daher die die im Rentenrecht für Zeiten bis 1972 existierende „Rente nach Mindesteinkommen“ für keine sogenannte „versicherungsfremde Leistung“ (1990, S. 288). Diese Argumentation spricht klar für eine Ausweitung dieser oder ähnlicher Regelungen im deutschen Rentensystem.

Der internationale Vergleich von Rentensystemen zeigt ebenfalls ganz eindeutig: Eine effektive Armutsvermeidung und Anerkennung von Lebensleistung setzt eine Aufwertung geringer Einkommen bei der Rentenberechnung voraus und zugleich impliziert sie eine Aufweichung des Subsidiaritätsprinzips für langjährig Versicherte. Eine Bedürftigkeitsprüfung darf bei ihnen nicht erfolgen, weil die jahrzehntelange Beitragsleistung in

8 Die OECD weist seit vielen Jahren regelmäßig in ihren Statistiken darauf hin, dass in Deutschland die Ersatzraten für Geringverdiener weit unterhalb des OECDDurchschnitts liegen. Vgl. OECD 2019, S. $143 \mathrm{ff}$. 
das Rentensystem, das für sehr viele Menschen das einzige Sicherungssystem zur Vermeidung von Altersarmut darstellt, dadurch fundamental entwertet würde. Die besondere Honorierung langjähriger Beitragszahlungen ist in jedem Versicherungssystem ein ubiquitäres Phänomen. Begründen lässt sie sich damit, dass langjährig Versicherte Personen mit ihren Beiträgen in besonderem Maße für die Stabilität des Systems gesorgt haben. Die eigentliche Alternative zu einer Aufwertung von Entgeltpunkten langjährig Versicherter mit geringem Verdienst ohne Bedürftigkeitsprüfung ist eine steuerfinanzierte Aufstockung niedriger Renten mit Bedürftigkeitsprüfung. Hier werden niedrige Renten - falls kein Vermögen bzw. anderes Einkommen zur Verfügung steht - auf ein bestimmtes Niveau aufgestockt und der Aufstockungsbetrag der Rentenversicherung aus Steuermitteln erstattet. Bereits Mitte der 1980er Jahre hatte der Berliner Sozialsenator Ulf Fink (CDU) eine solche Aufstockung der Renten - genannt „Ausgleichszulage“ - vorgeschlagen. Der damalige Bundesarbeitsminister Blüm lehnte diesen Vorschlag ab, weil er Sozialhilfe und Rentenversicherung zu einem „Mischsystem“ mache (vgl. Schmähl 2018, S. 801). Zudem gab es Bedenken, dass er dem Versicherungsgedanken entgegenstehe und die Gefahr bestehe, dass der Bund seinen Finanzierungsverpflichtungen nicht nachkomme, sodass diese bedürftigkeitsgeprüfte Leistung schließlich doch aus Beiträgen zu finanzieren wäre. ${ }^{9}$ Gegen eine solche Aufstockung der Rente nach Bedürftigkeitsprüfung spricht nicht zuletzt - wie Maydell ausführt aus verfassungsrechtlicher Sicht der „sozialstaatliche Handlungsauftrag“, demgemäß das staatliche Rentensystem für den Großteil der Versicherten „den Lebensbedarf sichernde Leistungen“ gewährleisten muss. Die Sozialhilfe sollte nur ,im Einzelfall eintreten, sie hat jedoch nicht die Funktion, die Regelsicherung für ganze Personengruppen zu gewährleisten“ (Maydell 1990, S. 416). Diese Argumentation mag nicht für eine Grundrente in Kombination mit einer Einkommensprüfung - also einer Art von „Bedürftigkeitsprüfung light“ - gelten, wie sie nach aktuellen Plänen von der Bundesregierung für das Jahr 2021 vorgesehen ist. Allerdings droht eine solche

9 In diesem Sinne formuliert Kolb (1990, S. 210f.): „Wie rasch ließe sich argumentieren, das Rentenniveau sei reduzierbar, weil kleinere Renten, die davon am stärksten betroffen wären, durch die Mindestsicherung aufgefangen würden. Ferner könnte die Gefahr bestehen, dass der Gesetzgeber bei knappen Haushaltsmitteln die Kosten für eine solche Mindestsicherung auf die Rentenversicherung überwälzt. Als das geeignetere Instrument erscheint deshalb die - auch für Zeiten über 1991 hinaus verlängerte - Rente nach Mindesteinkommen. Sie sichert die der Rentenversicherung mindestens 35 Jahr lang angehörenden Person deutlich über dem durchschnittlichen Sozialhilfeniveau." 
Einkommensprüfung nach und nach - ähnlich wie dies bei der Einkommensanrechnung der Hinterbliebenenrenten zu beobachten ist - zu einer umfänglichen Bedürftigkeitsprüfung zu mutieren, weil immer weitere Einkommensbestandteile bei der Anrechnung Berücksichtigung finden.

Die renten politische Grundfrage lautet heute also (wieder einmal): Soll die gesetzliche Rentenversicherung Mindestsicherungselemente stärken, oder soll eine bedürftigkeitsgeprüfte Mindestsicherung innerhalb des Rentensystems eingeführt werden? Letzteres, wie auch die vielfach geforderte Ausweitung der Freibeträge auf Renteneinkommen im Falle des Grundsicherungsbezugs, würde das staatliche Rentensystem im Ergebnis allerdings nicht stärken, sondern weiter schwächen, weil der oben erwähnte Prozess der „systemischen Verschmelzung“ dadurch nicht aufgehalten würde. Sozialstaatliche Vorsorge sollte ermöglichen, die Bürger in ein möglichst hohes gesetzliches Sicherungsniveau einzubinden, um ihnen einen angemessenen Lebensstandard auch im Alter zu gewährleisten. Hierzu sind neben einer breiten und ausreichenden Aufstockung niedriger Einkommen bei der Rentenberechnung vor allem ein ausreichend hohes Rentenniveau im Sinne einer „strukturellen Armutsfestigkeit“ (Dedring et al. 2010) des Rentensystems notwendig.

\section{Fazit}

In welche Richtung sollten notwendige Strukturreformen innerhalb des Rentensystems wirken? Zur Beantwortung dieser Fragen, lohnt sich ein Blick auf die Spezifika des deutschen Rentensystems. Anders als in den meisten anderen OECD-Staaten sind die öffentlichen Ausgaben für die Alterssicherung in Deutschland (gemessen als Anteil am BIP) in den Jahren 2000-2015 nicht gestiegen, sondern zurückgegangen (vgl. OECD 2019, S. 199). Dies ist - abgesehen von der sukzessiven Absenkung des Rentenbeitrags seit 2010 - ein Indiz für die Unterfinanzierung des staatlichen Rentensystems. In Deutschland ist - vielleicht weil der Rentenbeitragssatz aufgrund der Leistungskürzungen im Rentensystem und der teilweise guten Arbeitsmarktlage - seit Jahren eine sinkende Tendenz aufweist - diese einfache Tatsache noch immer nicht angekommen: In einer Gesellschaft, die altert, müssen die Kosten der Absicherung des Langlebigkeits- und Invaliditätsrisikos zwangsläufig steigen. Eine wichtige Aufgabe der Rentenpolitik besteht deshalb darin, für diese Tatsache Akzeptanz in der Gesellschaft zu schaffen. Die jüngst von der GroKo beschlossene Garantie, dass der Rentenbeitragssatz bis 2025 nicht weiter sinken darf, ist daher ein Schritt in die richtige Richtung. Eine künftige Rentenreform muss vor al- 
lem an den spezifischen Schwächen des deutschen Rentensystems ansetzen und folgende Elemente enthalten: Eine ausreichend hohes Ersatzrate für langjährig Versicherte, deutlich höhere Ersatzraten für Geringverdiener sowie eine Stärkung der sozialen Ausgleichselemente im Rentensystem. Das Rentensystem stellt den arbeitenden Menschen im Alter rechtlich besser als den Fürsorgeempfänger. Und es soll vor allem der Vermeidung von Armut dienen, wenn die Arbeitskraft im Alter oder im Falle von Invalidität erloschen ist. Was das Invaliditätsrisiko betrifft, ist die Lage heute nicht viel besser als vor 130 Jahren. 2018 lag der durchschnittliche Zahlbetrag einer Erwerbsminderungsrente in Deutschland bei 735 Euro (DRV Bund 2019, S. 120). Das waren rd. $24 \%$ des verfügbaren durchschnittlichen Bruttolohns. 1890 lag dieser Wert bei $20 \%$.

\section{Literatur}

Bremer Institut für Arbeitsmarktforschung und Jugendberufshilfe (2019): Armutsgefährdungsquoten im Ländervergleich: Rentner-innen, 65 Jahre und älter 2005-2018 (Mikrozensus), http://www.biaj.de/archiv-materialien/1301-armutsge faehrdungsquoten-im-laendervergleich-rentner-innen-65-jahre-und-aelter-2005-2 018-mikrozensus.html (abgerufen am 28.11.2019)

Blank, F./Logeay, C./Türk, E./Wöss, Josef/Zwiener, R. (2016): Alterssicherung in Deutschland und Österreich: Vom Nachbarn lernen? WSI Report 27, Düsseldorf

Dedring, K.-H./Deml. J./Döring, D./Steffen, J./Zwiener, R. (2010): Rückkehr zur lebensstandardsichernden und armutsfesten Rente, WISO Diskurs, August 2010, Bonn

DRV Bund (2019), Rentenversicherung in Zahlen 2019, Berlin

DRV Bund (2020): Internetseite der LeA-Studie (Lebensverläufe und Altersvorsorge), https:/www.lea-studie.de (abgerufen am 31.01.2020)

Eichenhofer, E. (2019): Grundrente und EU-Recht, in: Zeitschrift für europäisches Sozial- und Arbeitsrecht 9/2019, S. 359 - 364

Göckenjan G. (2000): Das Alter würdigen, Frankfurt am Main

Haan, P./Stichnoth, H./Blömer, M./Buslei, H./Geyer, J./Krolage, C./Müller, KaiUwe (2017): Entwicklung der Altersarmut bis 2036 - Trends, Risikogruppen und Politikszenarien, Gütersloh

Haan, P./Kemptner, D./Lüthen, H. (2019): Besserverdienende profitieren in der Rentenversicherung zunehmend von höherer Lebenserwartung, in: DIW Wochenbericht 23/2019, S. 392-399

Himmelreicher, R. K. (2018): Die Entwicklung und Verteilung von Altersrenten in Ost- und Westdeutschland, in: Datenreport 2018, Bonn, S. 333-339 
Hockerts, H. G. (1990): Entwicklungslinien der 100jährigen Geschichte der gesetzlichen Rentenversicherung. Die Rentenreform 1957, in: VDR/Ruland, F. (Hrsg.): Handbuch der gesetzlichen Rentenversicherung, Neuwied, S. 93-104

Köhler-Rama, T. (2019): Zur Debatte über die Grundrente: Ist die Kritik berechtigt?, in: Soziale Sicherheit 4/2019, S. 166-170

Kolb, R. (1990): Zukunftsperspektiven der Rentenversicherung, in: VDR/Ruland, F. (Hrsg.): Handbuch der gesetzlichen Rentenversicherung, Neuwied, S. 189222

Maydell, B. (1990): Die Rentenversicherung als Teil der öffentlichen Alterssicherung, in: VDR/Ruland, F. (Hrsg.): Handbuch der gesetzlichen Rentenversicherung, Neuwied, S. 411-423

Rolfs, C. (2000): Das Versicherungsprinzip im Sozialversicherungsrecht, München

Ruland, F. (2012): Grundprinzipien des Rentenversicherungsrechts, in: Eichenhofer, E./Rische, H./Schmähl, W. (Hrsg.): Handbuch der gesetzlichen Rentenversicherung SGB VI, Köln, S. 341-381

Schmähl, W. (2018): Alterssicherungspolitik in Deutschland, Tübingen

Steffen, J. (2019): Die Entwicklung von Grundsicherung und Altersrente seit 2003, Ein Prozess systemischer Verschmelzung, http://www.portal-sozialpolitik.de/ind ex.php?page $=$ grundsicherung-altersrente-verschmelzung (aufgerufen am 28.11.2019)

Torp, C. (2019): Mindestsicherungselemente in der deutschen Alterssicherung von Bismarck bis Merkel, in: DRV 74 (2), S. 132-149

OECD, Pensions at a Glance 2019, OECD and G20 Indicators, Paris

Wallerath, M. (1990), Rentenversicherung und Verfassungsrecht, in: VDR/Ruland, F. (Hrsg.): Handbuch der Rentenversicherung, Neuwied, S. 288-323

Wallrabenstein, A. (2009): Versicherung im Sozialstaat, Tübingen 
